Aigerim Altayeva, $\mathrm{PhD}$ student International University of Information Technologies

Almaty, the Republic of Kazakhstan

E-mail: aikosha1703@gmail.com

ORCID ID 0000-0002-9802-9076

\title{
MULTI-AGENT BASED MICROCLIMATE CONTROL IN RESIDENTIAL BUILDINGS
}

\begin{abstract}
The methodology of system design of modern intelligent control systems in the electric power industry and their features are considered when providing the required comfort in multi-zone buildings with the use of multi-agent power consumption and comfort management systems. This control system covers all the monitored zones of the building and, if necessary, allows providing the greatest possible overall comfort in the building while reducing the available electric power.
\end{abstract}

\section{Key words: HVAC System, Thermal Process, Indoor Microclimate.}

The energy consumption problem began to appear projects of buildings for which the main source of electricity is renewable energy, the so-called building zero energy balance. A constant level of comfort or permanent production is one of the most important in the world today. To solve this problem, energy renewable sources (solar panels, wind generators) are used. Constructions of buildings lead to a decrease in costs of electricity and further reduce.

1) The loads on the power grid.

2) Emergency situations.

Energy consumption in the sector of construction and operation of buildings is large enough. Currently, the world pays great attention to the problems of conservation and the efficient use of energy buildings. It should be remembered about the impact of indoor environment on health and well-being of people, as in buildings, people spends most of their lives. The problem of energy efficiency and comfort in buildings can be solved by the control systems. However, currently, there are no well-established approaches and recommendations for the development of energy management systems and comfort in buildings. The existence of such control systems is especially important for buildings that are powered by self-contained renewable electricity (RES) or from sources with limited power.

Part of the above problem can be solved by dividing the building into zones, and climate control in the zones $[1,2,3]$. Each room can be a separate zone; it is also possible to combine several rooms in the same zone if they have similar conditions. Sometimes one room can be divided into several zones, if the environmental conditions they are not the same and should be set independently.

Comfort in a building or areas generally defined by the combination of temperature, illumination, $\mathrm{CO}_{2}$ concentration. In some cases, consider when evaluating the humidity comfort, air velocity and some other parameters. Sometimes using the projected average rating instead of the air temperature, this shows the predicted human sensitivity to thermal conditions. This estimate depends on the parameters of the environment (air temperature, mean radiant heat, speed and air humidity), physical activity and clothing. The necessary conditions for the implementation of an artificial agent certain behaviors serve special devices directly perceive the external environment (receptors) and executive bodies, impact on the environment (effectors), and the processor - a block of information processing and memory. In theory, MAC is believed that one agent has only 
a partial view of the global problem, which means that it can only solve some of the common tasks. In this regard, to address the complex problem it is necessary to create a set of agents and to organize the effective interaction between them, which will build a single multi-agent system. In multi-agent systems, the whole range of tasks according to certain rules is distributed among all agents, each of which is considered to be a member of an organization or group. The distribution of tasks is assigning each agent of a role, the complexity of which is determined on the basis of possible agents. In accordance with the theory of multi-agent systems, agent represented in the form:

$$
A g=<G, S, A, \theta, \varphi>
$$

where, G-the objective function, $S=\left\{S_{1}, s_{2}, \ldots, s_{n}\right\}$ is a set of actions, knowledge base $\theta$ and operation $\varphi: G \times \theta \times S \rightarrow A$.

In our case we defined the following set of $A g_{I}=<A g_{e c}, A g_{e p}, A g_{m}>$

where, $A g_{e c}-$ Agent of energy consumption, $A g_{e p}$ - Agent of energy production, $A g_{m}-$ Agent of modeling.

Agent of energy consumption: the main purpose of this agent is to collect information on energy consumption data and transmit them to the modeling agent.

Agent of energy production: the main purpose of the agent is handling the technical data of the system and transfers the results of the simulation processing agent. Agent modeling: the main function of this agent is to build predictive values of consumption and production on the basis of data received from agents $A g_{e c}$ and $A g_{e p}$ performance hybrid power system. In the simulation, modeling agent uses the data received from the agent of energy and builds on the basis of these data, forecast, using the average forecasting model, according to which the consumption forecast at time $\mathrm{t}(\mathrm{W} \mathrm{h})$ is defined as:

$$
\hat{y}_{t}=\sum_{i=1}^{p} y_{t-L i}
$$

where, $\mathrm{p}$ - the order of the equation, L - Parameter lag.

Agent electricity production model is based on empirical nominal solar panel production estimates on different days and expressed by the following formula:

$$
\hat{z_{t}}=k_{t} x_{t}
$$

where, the coefficient $k$ is a function of the number of the month. Depending on the month of manufacture is calculated in the average length of time it and solar power panel.

Current trends for the management and control of electric power systems are moving towards the use of intelligent agent technologies, which are known as multi-agent systems [3]. A multi-agent system is a combination of several agents that interact with each other to achieve the common goal of a control system. 


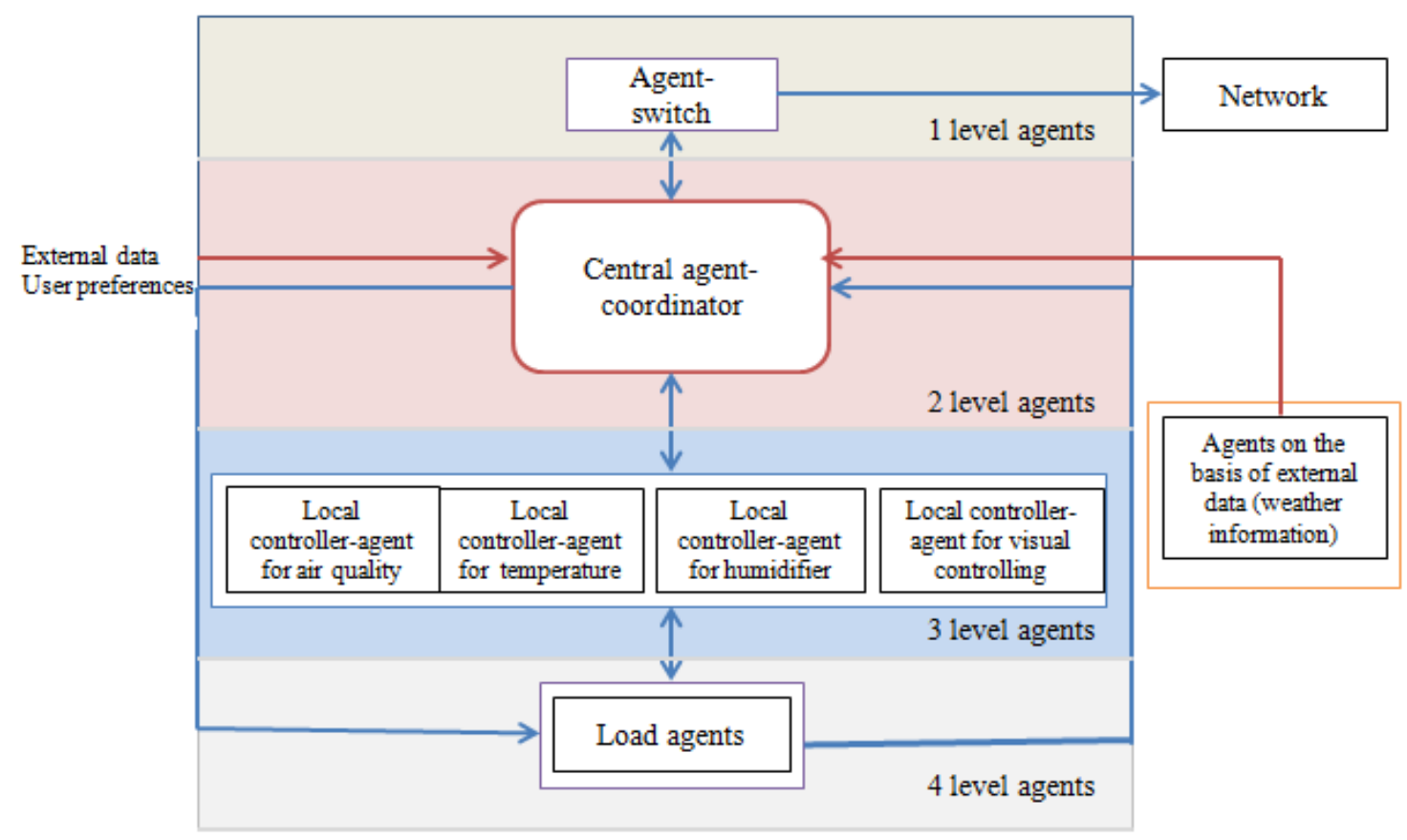

Figure 1 - Multi-agent control system for energy-efficient buildings

Figure 1 show only the functional elements of the control system, without the power circuits of various loads, run management agents [4, 5, 6]. All agents are classified into four levels, depending on the function and purpose. Agent-switch, the agent coordinator, local and load agents.

1) Agent switch located on the first level. Agent-switch receives the data from the central coordinator of the consumed and generated energy.

2) Central agent coordinator, which is located on the second level, is one of the key elements in the control system. It interacts with all of agents on the basis of external data (weather information) and user preferences on thermal, visual comfort and air quality, as well as on the basis of the electrical loads of the building data.

3) For monitoring thermal and visual comfort, air quality controller uses four local agents. These agents controller compares the current values of comfort parameters set and the amount of energy needed to control the actuators of engineering systems of the building.

4) Load agents generally responsible for the whole process. The interrelationship between all agents and for the final result of all its processes in the supply of energy.

Power management system and comfort should cover all areas of the building and allow optimizing the distribution of electric energy in case of reduction of the available electric power. Every area of the building must be set a weighting (priority) that is accounted for in the optimization of energy consumption. In the comfort of the management model is needed comfort, which will reflect the most required conditions and user preferences. Optimization of distribution of electric energy in the zones of the building can be performed by a cluster of particles. When, optimizing model is needed in the conversion of electrical energy required comfort conditions [7].

Maintaining maximum comfort in the building is possible with temperature control, humidity, lighting and $\mathrm{CO}_{2}$ levels. These parameters are managed by individual areas of the building. Comfort in each zone has its own weight in total comfort. Comfort control system shall cover all areas of the building and allow optimizing the distribution of electric energy in case of reduction of the available electric power. 


\section{REFERENCES}

1. Frey, S., Diaconescu, A., Menga, D., Demeure, I.: A holonic control architecture for a heterogeneous multi-objective smart micro-grid. In: IEEE 7th International Conference on Self-Adaptive and Self-Organizing Systems (SASO). (Sept 2017) 21-30.

2. Wyon, D. P. W. P., 2013. How Indoor Environment Affects Performance. ASHRAE Journal, 55 (3), pp. 46-52.

3. Lee, Y. S. \& Malkawi, A. M., 2014. Simulating multiple occupant behaviors in buildings: An agent-based modeling approach. Energy and Buildings, 69, pp. 407-416.

4. Langevin, J., Wen, J. \& Gurian, P. L., 2015. Simulating the human-building interaction: Development and validation of an agent-based model of office occupant behaviors. Building and Environment, 88, pp. 27-45.

5. Langevin, J., Wen, J. \& Gurian, P. L., Including occupants in building performance simulation: Integration of an agent-based occupant behavior algorithm with EnergyPlus. In: ASHRAE/IBPSA, ed. 2014 ASHRAE/IBPSA-USA Buidling Simulation Conference. Atlanta, GA, Sept 10-12 2016.

6. L. Neumeyer, B. Robbins, A. Nair, and A. Kesari, "S4: Distributed Stream Computing Platform," 2010 IEEE International Conference on Data Mining Workshops, pp. 170-177, 2015.

7. Carbo and J. M. Molina, "Agent-based collaborative filtering based on fuzzy recommendations," International Journal of Web Engineering and Technology, vol. 1, no. 4, p. 414, 2014. 